\title{
UMA ABORDAGEM PARA O DESENVOLVIMENTO DE APLICAÇÕES NO CUIDADO DE SAÚDE PERVASIVO ATRAVÉS DO USO DE ARQUÉTIPOS
}

\author{
An Approach to Developing Applications in the Pervasive Healthcare Environment \\ Through the Use of Archetypes

\begin{abstract}
João Luís Cardoso de Moraes'; Wanderley Lopes de Souza²; Luís Ferreira Pires³; Luciana Tricai Cavaliniं; Antônio Francisco do Prado²
\end{abstract}

Resumo Objetivos: Este trabalho propõe uma abordagem para o desenvolvimento de aplicações no ambiente de Cuidado de Saúde Pervasivo, através do uso de arquétipos. Materiais e Métodos: O Cuidado de Saúde Pervasivo foca o uso de novas tecnologias, ferramentas e serviços, para ajudar os pacientes a ter um papel mais ativo no tratamento de suas condições. Ambientes de Cuidado de Saúde Pervasivo exigem uma grande de troca de informações, e tecnologias específicas têm sido propostas para prover interoperabilidade entre os sistemas que compõem tais ambientes. No entanto, a complexidade dessas tecnologias faz com que seja difícil adotá-las amplamente, bem como migrar Ambientes de Cuidado de Saúde centralizados para Ambientes de Cuidado de Saúde Pervasivo. Resultados: A abordagem foi demonstrada e avaliada em um experimento controlado realizado no departamento de cardiologia de um hospital localizado na cidade de Marília (São Paulo, Brasil). Conclusão: Um aplicativo foi desenvolvido para avaliar a abordagem, e os resultados mostraram que a abordagem é adequada para facilitar o desenvolvimento de sistemas de cuidado de saúde, oferecendo soluções de abordagem genéricas e poderosas.

Palavras-chave: Cuidado de Saúde Pervasivo, Computação Ubíqua, openEHR, Arquétipos, Linguagem Específica de Domínio

Abstract Aims: This paper proposes an approach to develop applications in the Pervasive Healthcare environment, through the use of Archetypes. Materials and Methods: Pervasive Healthcare focuses on the use of new technologies, tools, and services, to help patients to play a more active role in the treatment of their conditions. Pervasive Healthcare environments demand a huge amount of information exchange, and specific technologies have been proposed to provide interoperability between the systems that comprise such environments. However, the complexity of these technologies makes it difficult to fully adopt them and to migrate Centered Healthcare Environments to Pervasive Healthcare Environments. Results: This approach was demonstrated and evaluated in a controlled experiment that we conducted in the cardiology department of a hospital located in the city of Marilia (São Paulo, Brazil). Conclusion: An application was developed to evaluate this approach, and the results showed that the approach is suitable for facilitating the development of healthcare systems by offering generic and powerful approach capabilities.

Keywords: Pervasive Healthcare, Ubiquitous Computing, openEHR, Archetypes, Domain Specific Language.

1. Pós-graduando da Universidade Federal de São Carlos; 2. Professor da Universidade Federal de São Carlos; 3. Professor at University of Twente - PhD; 4. Professor da Universidade Federal Fluminense; 


\section{Introdução}

A maioria dos países enfrenta muitos problemas relacionados à saúde, tais como cuidados de saúde caros e a baixa qualidade dos serviços de saúde prestados. Esses problemas começaram a aparecer como resultado do aumento da população e da falta de profissionais de saúde, que se agrava devido ao número crescente de idosos, mais doenças crônicas e a crescente demanda por novos tratamentos de saúde e tecnologias. Por exemplo, nos Estados Unidos, o número de médicos formados em 1981 foi de 15.632 e de 15.712 em 2001 (aumento de apenas 0,5\%), enquanto no mesmo período sua população aumentou de 1 milhão de 226 (aumento de 24\%)'.

O atual modelo de cuidado de saúde está centrado em pessoas altamente especializadas, localizadas em hospitais de grande porte e com foco em casos agudos para tratamento de pacientes, mas há a necessidade de mudar para um modelo de saúde distribuído, a fim de produzir respostas mais rápidas e permitir que os pacientes possam melhor gerenciar sua própria saúde. O modelo centralizado de saúde implica que os pacientes e cuidadores de saúde tenham que visitar a mesma instituição de saúde (um hospital ou clínica) para prover serviços de saúde, que muitas vezes são caros e ineficientes. Um modelo de saúde distribuído, que pervada a vida quotidiana dos cidadãos, é mais capaz de fornecer cuidados de saúde mais baratos, mais eficazes e oportunos, caracterizando o Cuidado de Saúde Pervasivo. De acordo com², o objetivo do Cuidado de Saúde Pervasivo é permitir o gerenciamento do cuidado saúde e bem-estar dos pacientes, usando tecnologias de informação e comunicação para disponibilizar cuidados de saúde em qualquer lugar, a qualquer momento e para qualquer pessoa.

A Computação Ubíqua $^{3}$ tem sido considerada a nova era da computação e tem como objetivo per- mitir que o usuário acesse as informações facilmente de qualquer lugar, a qualquer momento e usando qualquer tipo de dispositivo. Ambientes de Computação Ubíqua, seja em comunidades, lares ou hospitais, podem ser extremamente úteis para construir um modelo de Cuidado de Saúde Pervasivo. Particularmente, para que a "alma" deste modelo seja voltada para o paciente, um cuidado de saúde eficiente é fundamental. Desta forma, é necessário que a troca de informações entre os vários profissionais de saúde envolvidos no cuidado de saúde do paciente seja rápi$\mathrm{da}$, eficiente e segura.

A troca de informações entre sistemas heterogêneos de Registro Eletrônico de Saúde (RES) em ambientes de Cuidado de Saúde Pervasivo requer padrões de comunicação que permitam a interoperabilidade entre esses sistemas. Embora o Health Level Seven $(H L 7)^{[1]}$ seja um padrão internacional utilizado para troca de mensagens entre Sistemas Informação Saúde (SIS) heterogêneos, ele apresenta limitações conhecidas por representar conhecimento clínico, tais como o uso combinado de componentes estruturados e termos codificados, que podem resultar em interpretações inconsistentes de informação clínica ${ }^{4}$. O openEHR ${ }^{[2]}$ é uma fundação dedicada à pesquisa em RES interoperável, a qual definiu uma arquitetura aberta baseada em modelagem de dois níveis que separa as informações de conhecimentos, portanto tratando algumas das limitações do HL7.

Este trabalho propõe uma abordagem de desenvolvimento de aplicativos no ambiente de Cuidado de Saúde Pervasivo, usando arquétipos e linguagens específicas de domínio (DSLs)5. DSLs permitem a criação de uma infraestrutura para reuso e geração de código das aplicações de um domínio alvo. Este artigo está estruturado da seguinte forma: a Seção 2 apresenta os materiais e métodos utilizados neste artigo, descrevendo os principais conceitos sobre o padrão de saúde 
openEHR, os conceitos de linguagens específicas de domínio e a abordagem proposta, ilustrada com um estudo de caso no domínio da saúde. A Seção 3 apresenta os resultados e discute a avaliação do aplicativo desenvolvido usando a abordagem proposta. A Seção 4 discute alguns trabalhos relacionados. Finalmente, a seção 5 apresenta as conclusões e propõe a continuação dos trabalhos.

\section{Materiais e Métodos}

\section{1 - OpenEHR Dual Model}

A arquitetura openEHR foi desenvolvida com base no paradigma de modelagem de dois níveis, conforme mostrado na Fig. 1. No primeiro nível, um Modelo de Referência (RM) foi definido como um conjunto predefinido de classes que modelam a estrutura de um registro eletrônico de saúde; e no segundo nível, definiram-se conceitos específicos, restringindo as classes do RM em termos de arquétipos, expressos em Linguagem de Definição de Arquétipo (ADL) .

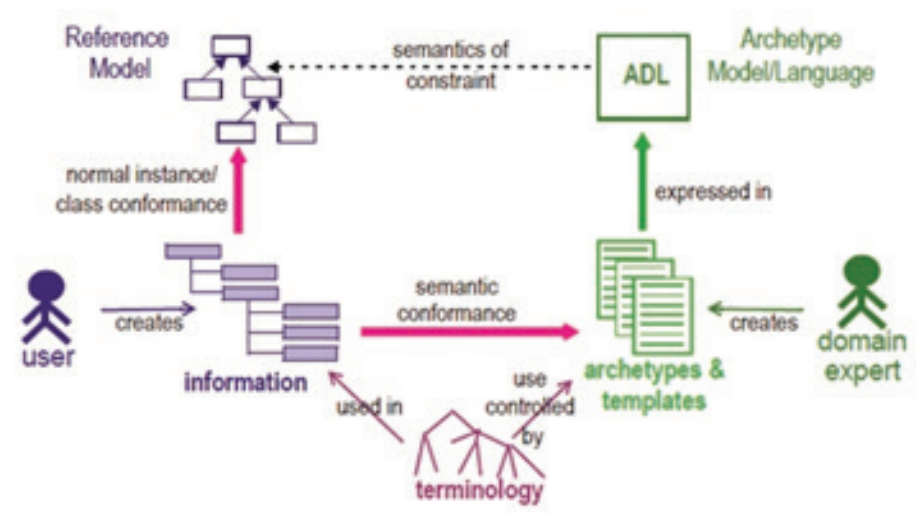

Figura 1. Modelo Dual openEHR

Um arquétipo é um modelo formal de um conceito de domínio e se espera que seja facilmente compreensível por um especialista do domínio. No nível de implementação arquétipos podem ser traduzidos para qualquer idioma. Em conformidade com a modelagem de dois níveis, dados de entrada são armazenados de acordo com o RM, mas também devem respeitar os conceitos expressos pelos arquétipos. Os arquétipos são projetados por especialistas do domínio e não por profissionais de tecnologias de informação. Esta separação deve facilitar a interpretação do conhecimento extraído das mensagens trocadas pelos sistemas de saúde em várias aplicações.

No Modelo de Referência openEHR, uma classe COMPOSITION refere-se a uma ou mais instâncias de uma classe SECTION que contém objetos da classe ENTRY. A classe ENTRY representa o conteúdo de um registro clínico durante uma Observação, Exame, Avaliação, ou Intervenção em um paciente. ENTRY é definida como um tipo abstrato com quatro subtipos concretos: (1) OBSERVATION que pode ser utilizado para representar observações clínicas, como pressão sanguínea; (2) EVALUATION que pode ser utilizada para representar avaliações feitas depois que uma observação clínica for concluída, como risco de avaliação do paciente; e (3) INSTRUCTION e ACTION, que podem ser utilizadas para representar procedimentos cirúrgicos, medicações e outras intervenções clínicas. A classe ACTION descreve o que foi feito e registra no histórico do RES do paciente como resultado de uma INSTRUCTION.

O Gerenciador de Conhecimento Clínico - Clinical Knowledge Manager (CKM) é um repositório de arquétipos proposto pela fundação openEHR. Este repositório contém um conjunto de arquétipos que representam conceitos clínicos e podem ser reutilizados em várias aplicações do domínio de saúde. Neste projeto foram reutilizados alguns arquétipos providos pelo CKM, como Device, Device Details e Clinical Synopsis, mas também foram desenvolvidos novos arquétipos para representar conceitos clínicos do domínio de cardiologia, como Pacemaker Implantation, Vascular Cardiac Surgery, Coronary Cardiac Surgery, Angioplasty Cardiac e Pacemaker Evaluation. 
Os arquétipos openEHR foram definidos para uso genérico, podendo definir qualquer linguagem ou terminologia. Um arquétipo consiste de três seções: cabeçalho, definição e ontologia. A Fig. 2 mostra um extrato de um arquétipo na linguagem $A D L$ para 0 conceito clínico de Implante de Marcapasso definido de acordo com o padrão openEHR. O cabeçalho inclui o nome do arquétipo (linha 1). A seção definição contém a estrutura e as restrições associadas ao conceito clínico definido pelo arquétipo. Pacemaker Implantation especializa a classe ACTION (linha 4) do Modelo de Referência. O CLUSTER (linha 8) refere-se ao modo de estimulação utilizado para o implante de um marcapasso e consiste de um ELEMENT com valor do tipo DV_TEXT. A seção ontologia (linha 14-20) inclui as definições terminológicas. No exemplo, a expressão linguística 'Pacemaker Implantation' está associada ao código ‘at0000' (linha 4).

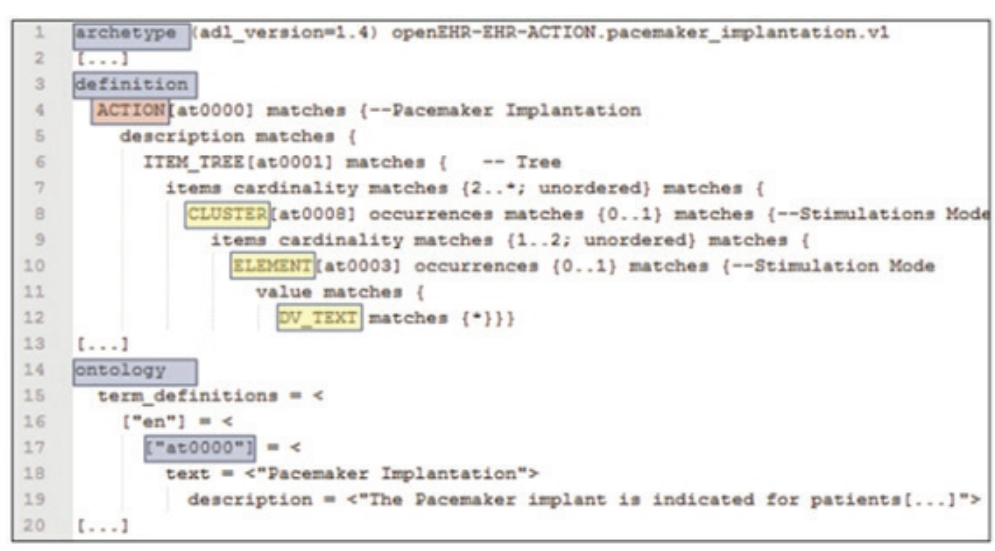

Figura 2. Arquétipo para Conceito Pacemaker Implantation

A abordagem de modelagem de dois níveis aplicada em openEHR permite que os engenheiros de software desenvolvam aplicações separadamente do domínio de modelagem, por meio da especialização e instanciação de classes do RM. Especialistas de domínio podem introduzir novos conceitos, definindo novos arquétipos, de modo que os sistemas de software não necessitem ser projetados e implantados novamente, sempre que um novo conceito for definido. Portanto, a abordagem de modelagem de dois níveis tende a tornar os sistemas mais fáceis de manter e estender com novas aplicações. Arquétipos desempenham um papel de gateways semânticos para terminologias, classificações e diretrizes clínicas informatizadas. Arquétipos foram introduzidos pela fundação openEHR para melhorar o nível de interoperabilidade semântica entre vários sistemas de informação de saúde.

\section{2 - Linguagem Específica de Domínio}

Uma DSL é uma linguagem projetada para ser útil para um conjunto específico de tarefas dentro de um determinado domínio. Ela pode ser definida por um metamodelo, que representa o conhecimento do domínio do problema. Restritas a um domínio específico, DSLs são geralmente pequenas, consistindo de apenas um conjunto de abstrações e notações, que são próximas de termos reais conhecidos por especialistas do domínio7. Assim, DSLs expressam soluções no nível de abstração do domínio do problema, reduzindo os esforços de tradução dos conceitos deste domínio para o domínio da solução.

O uso de DSLs na modelagem de aplicações, ao invés de linguagem de modelagem de propósito geral (por exemplo, Unified Modeling Language - UML), permite a criação de modelos mais específicos e mais completos. Recursos, tais como frameworks, padrões de projeto e componentes, podem ser incluídos nos modelos, criando uma infraestrutura que permite a execução de transformações de Modelo-para-Código (M2C), para a geração de uma quantidade maior de código a partir da modelagem.

\section{3 - Abordagem Proposta}

A abordagem utiliza a modelagem de dois níveis openEHR como uma metodologia orientada a modelo 
proposta para permitir a interoperabilidade semântica entre sistemas de informação heterogêneos. A abordagem combina arquétipos e modelos para criar interfaces de comunicação, usando ADL como a linguagem específica do domínio (DSL) que expressa o conhecimento do domínio da saúde.

Usando a notação Structured Analysis and Design Technique (SADT) ${ }^{8}$, a Fig. 3 mostra uma visão geral da abordagem proposta, que consiste em duas etapas: Engenharia de Domínio (ED) e Engenharia de Aplicação (EA). Em ED é desenvolvida uma DSL representada por um metamodelo do domínio da saúde, e são desenvolvidas transformações $\mathrm{M} 2 \mathrm{C}$ para a geração de código. Em AE, aplicações ubíquas são desenvolvidas por meio do reuso dos artefatos desenvolvidos na etapa de ED. O metamodelo é usado para suportar a modelagem da aplicação e as transformações M2C são reutilizadas para geração de código contendo mensagens openEHR usando o arquétipo.

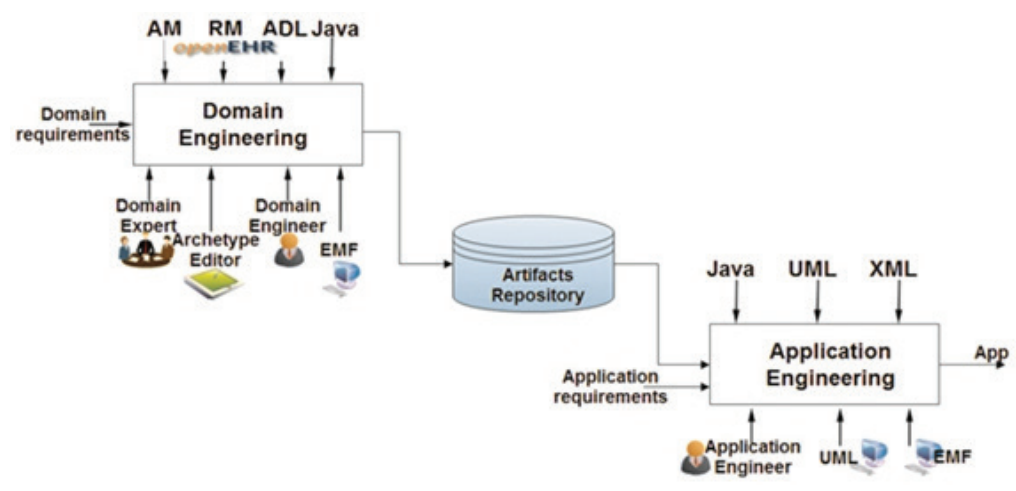

Figura 3. Visão Geral da Abordagem Proposta

\subsection{1 - Engenharia de Domínio}

A Fig. 4 ilustra as três atividades da etapa de Engenharia de Domínio: Especificar Metamodelo do Domínio, Projetar Metamodelo do Domínio e Implementar Metamodelo do Domínio.

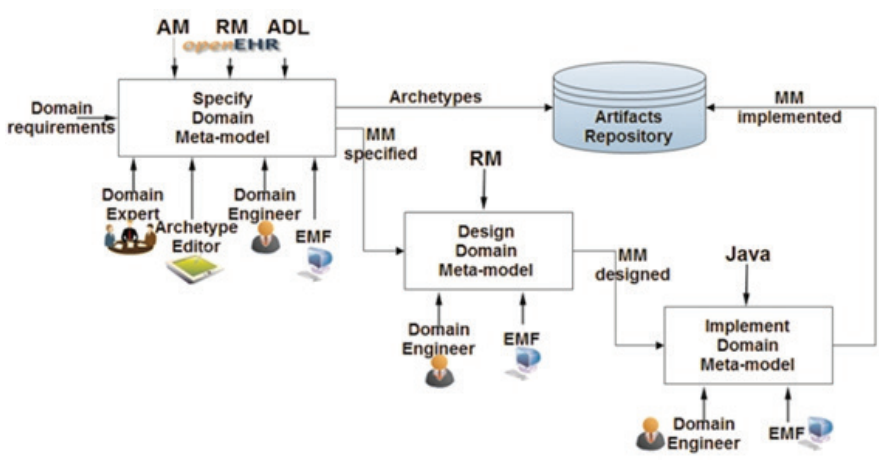

Figura 4. Engenharia de Domínio

Na atividade Especificar Metamodelo do Domínio, os requisitos de domínio de saúde são elicitados, especificados, analisados e representados em um metamodelo que expressa o conhecimento sobre este domínio. Usando o Eclipse Integrated Development Environment (IDE), o Engenheiro de Domínio, guiado pelas especificações do RM openEHR, analisa o metamodelo. O Modelo de Refêrencia openEHR representa as informações do domínio de saúde em um alto nível de abstração, que permite o uso de uma gama de terminologia na descrição de conceitos deste domínio. O Engenheiro de Domínio especifica os arquétipos, guiados por especificações do AM openEHR, pela linguagem $A D L$ e repositório CKM. Os Especialistas do Domínio e a ferramenta Archetype Editor ${ }^{[3]}$ são os principais mecanismos usados para suportar a especificação de arquétipos. As saídas desta atividade são os metamodelos do domínio, que representam os conceitos utilizados na modelagem do domínio e os arquétipos especificados.

Na atividade Projetar Metamodelo do Domínio, a especificação do metamodelo é refinada de acordo com padrões, tecnologias e plataformas que viabilizam a construção do metamodelo. O Engenheiro de Domínio, usando o Eclipse Modeling Framework (EMF), desenvolve a arquitetura do metamodelo do domínio, mostrando a decomposição funcional do domínio. O processo é guiado por uma codificação de terminologia médica, tal como 
Systematized Nomenclature of Medicine - Clinical Terms ${ }^{[4]}$ (SNOMED-CT). SNOMED-CT é um esquema de identificação de conceitos e termos clínicos e são usados por sistemas computacionais para suportar a interoperabilidade semântica. O metamodelo projetado possui uma estrutura flexível de modo que satisfaça todos os requisitos necessários e ainda possibilite um certo grau de liberdade para a sua implementação.

Na atividade Implementar Metamodelo do Domínio é realizada a implementação com base nas especificações contidas no metamodelo projetado. Apoiado pelo framework EMF e por um editor de modelos, o Engenheiro de Domínio gera automaticamente o código Java do metamodelo. O editor de modelos auxilia o Engenheiro de Domínio durante a especificação do modelo de aplicação, durante a etapa EA. O editor de modelo e o metamodelo estão disponíveis como um plugin no Eclipse IDE, a fim de que o metamodelo possa ser instanciado para a modelagem de aplicação durante EA. Uma vez que o metamodelo baseia-se no RM openEHR, os modelos criados usando a DSL, expressos por este metamodelo, também correspondem ao refinamento que deve ser realizado para criação de mensagens openEHR.

\subsection{2 - Engenharia de Aplicação}

A etapa EA envolve as disciplinas de Análise, Projeto e Implementação, que são parte do processo de desenvolvimento de software. A Fig. 5 mostra as três atividades da fase de Engenharia de Aplicação: Analisar Aplicação, Projetar Aplicação, e Implementar Aplicação.

O uso do DSL expresso por metamodelos do domínio de saúde para modelagem de aplicações na etapa EA, além de facilitar o desenvolvimento de aplicações para esse domínio, possibilita o mapeamento do processo de desenvolvimento de mensagens openEHR em ativi-

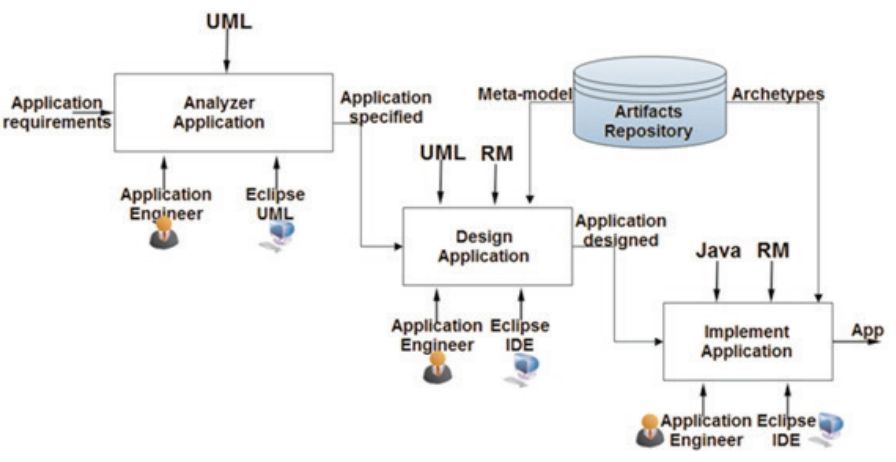

Figura 5. Engenharia de aplicação.

dades tradicionais da Engenharia de Software. Assim, o RM openEHR pode ser executado diretamente em modelos que especificam os requisitos das aplicações. Além disso, a geração de código automatiza muitas das tarefas de Engenheiro de Aplicação associadas à organização de mensagens openEHR, de acordo com os conceitos expressos pelos arquétipos. O metamodelo de cuidados de saúde também permite o reuso do conhecimento do domínio em vários projetos durante a etapa $E A$.

Para investigar a viabilidade da abordagem proposta, foi desenvolvido um estudo de caso, criando uma aplicação ubíqua que permite a avaliação de marcapasso implantado em pacientes. O paciente recebe uma notificação em seu dispositivo móvel para agendar uma entrevista para avaliação do marcapasso implantado. Quando o paciente chega à clínica, o médico recebe uma mensagem no seu dispositivo contendo informações relacionadas ao implante de marcapasso do paciente. As mensagens recebidas pelo médico e paciente são baseadas em arquétipos openEHR relacionados ao conceito de implante de marcapasso. As atividades da EA são detalhadas abaixo, apresentando exemplos, baseados na aplicação descrita como o estudo de caso.

Na atividade Analisar Aplicação, a aplicação é especificada de acordo com seus requisitos elicitados e analisados. Para a especificação, o Engenheiro de 
Aplicação utiliza técnicas já conhecidas da UML como diagramas de classe, sequência e casos de uso.

Na atividade Projetar Aplicação, a especificação da aplicação é refinada utilizando as tecnologias de plataformas de hardware e software, que permitem a implementação da aplicação, tais como Java Micro Edition (Java ME). Com base nos diagramas de caso de uso, nesta atividade, o Engenheiro de Aplicação também realiza a modelagem de termos e conceitos sobre o domínio do problema como uma instância de metamodelo construído na etapa ED, selecionando as classes e atributos que são relevantes para o domínio da aplicação.

A Fig. 6 mostra um modelo construído usando o plugin editor de modelo disponível na IDE Eclipse. Este modelo descreve as informações associadas às aplicações do domínio em conformidade com o arquétipo que representa o conceito de implante de marcapasso. A informação clínica é enviada para o médico, usando o padrão de saúde openEHR com arquétipos. A classe Pacemaker Implantation é um subtipo da classe ACTION do RM openEHR, conforme mostrado na Fig. 6' ${ }^{1} \mathrm{O}$ atributo stimulationMode representa o modo de estimulação usado durante a implantação do marcapasso, de acordo com o nó at0003 no arquétipo de Pacemaker Implantation, conforme mostrado na Fig. 6 (2).

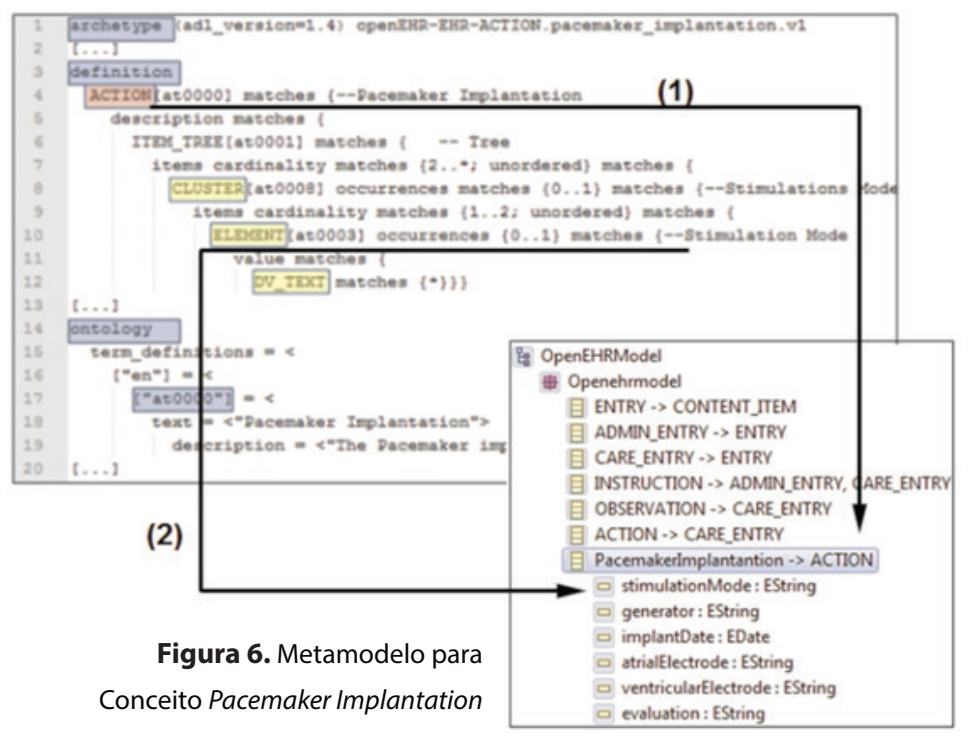

Adotando a terminologia usada no metamodelo (SNOMED-CT), o Engenheiro de Aplicação combina os arquétipos que definem os conceitos clínicos representados nos modelos usando o código SNOMED-CT correspondente. Por exemplo, a Fig. 6 mostra o atributo stimulationMode da classe Pacemakerlmplantation, no qual foi aplicado o código SNOMED-CT que identifica o conceito de implante de marcapasso na seção de ontologia do arquétipo. Assim, a aplicação torna-se capaz de transmitir dados clínicos compreensíveis de um modo interoperável.

Na atividade Implementação da Aplicação, a aplicação é implementada, incluindo sua interface de comunicação, através da integração dos arquétipos às mensagens openEHR. No Eclipse IDE, o Engenheiro de Aplicação é assistido pelo framework JET para realizar as transformações $\mathrm{M} 2 \mathrm{C}$ para geração parcial de código. Esse código gerado trata as mensagens openEHR usando arquétipos, as quais conterão os dados relacionados ao implante de marcapasso, de acordo com as especificações contidas no modelo. Após a geração parcial do código, o Engenheiro de Aplicação adiciona novos códigos quando necessário. Além disso, os outros componentes que compõem o aplicativo são desenvolvidos, tais como as interfaces do usuário e mecanismos para persistência de dados. No final da implementação, os testes são realizados para fornecer o feedback indicando se é necessário ou não retornar às atividades anteriores de etapa EA. A Fig. 7 mostra o arquétipo Pacemaker_ Implantation e a interface de usuário que contêm informações clínicas relacionadas com o estudo de caso.

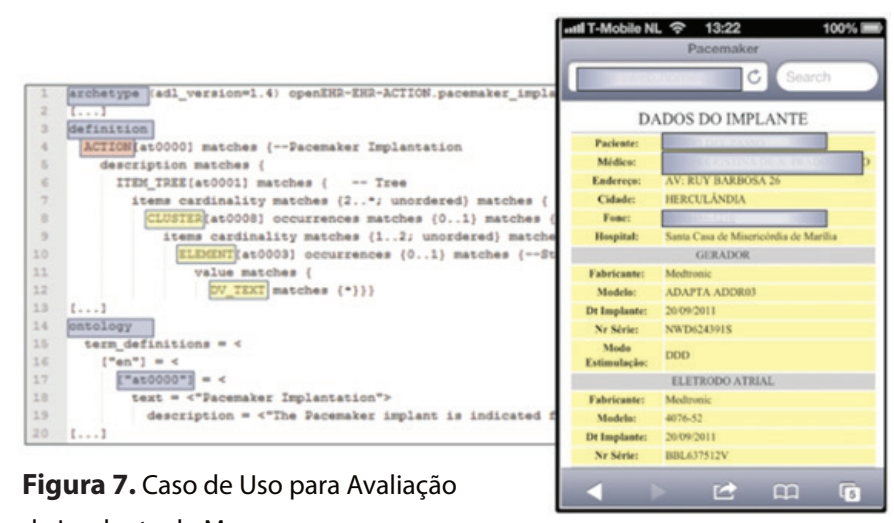

de Implante de Marcapasso 


\section{Resultados e Discussão}

Foi conduzido um experimento controlado ${ }^{9}$ no Departamento de Cardiologia do Hospital e Santa Casa de Marília (São Paulo), usando o cenário discutido na etapa de Engenharia da Aplicação da abordagem proposta. A Tabela 1 mostra o número de participantes do estudo de caso e a avaliação foi feita no período de 1 de Janeiro de 2012 a 30 julho de 2012.

\begin{tabular}{|l|c|}
\hline Participantes & $\begin{array}{c}\text { Cenário: } \\
\text { Avaliação de Implante } \\
\text { de Marcapasso }\end{array}$ \\
\hline Médicos & 3 \\
\hline $\begin{array}{l}\text { Estudantes de Medicina } \\
\text { (Residentes) }\end{array}$ & 2 \\
\hline Enfermeiras & 2 \\
\hline Pacientes & 15 \\
\hline Total & $\mathbf{2 2}$ \\
\hline
\end{tabular}

Tabela 1. Participantes (respondentes).

Neste estudo de caso foi utilizado o Modelo de Aceitação de Tecnologias $\left(\right.$ TAM $^{10}$, o qual assume que a Utilidade Percebida (PU) e Facilidade de Uso Percebida (PEU) podem predizer as atitudes favoráveis ao uso e Intenções para Usar determinada tecnologia (IU). TAM é conhecido por ser um modelo adequado para explicar o processo de aceitação de tecnologia no setor de saúde ${ }^{11}$. Para testar o efeito na aceitação de tecnologia, dois grupos foram selecionados para análise, envolvendo um conjunto limitado, mas relevante de pessoas: pacientes e cuidadores (estudantes de medicina, médicos e enfermeiras). Decidimos agrupar os cuidadores porque eles trabalham como uma equipe, no presente cenário.

Após a implementação do caso de uso, um questionário estruturado com

Tabela 2. Estatística e Confiabilidade dos Construtos. base no modelo TAM foi enviado aos cuidadores de saúde envolvidos no cenário, e um questionário também foi distribuído aos pacientes após o procedimento médico. Os entrevistados foram convidados a indicar a sua concordância ou discordância relacionada a várias questões em uma escala de cinco pontos de Likert ${ }^{12}$, variando de 1 = discordo totalmente a $5=$ concordo fortemente. Muitos estudos empíricos demonstraram que as propriedades psicométricas das escalas de medição podem ser afetadas pela ordenação dos itens dentro do questionário. Para evitar este viés, as questões foram misturadas aleatoriamente por meio dos construtos (PU, PEU, IU) e foi realizado um pré-teste com um grupo para garantir que as escalas estavam adequadas. Cerca de $80 \%$ e $73 \%$ dos questionários distribuídos aos pacientes e cuidadores respectivamente foram concluídas.

A análise consistiu em duas partes: (1), foram testadas a validade e confiabilidade do modelo de medição usando alfa de $\mathrm{Cronbach}^{13}$; (2) os dados foram analisados usando Structural Equation Modeling (SEM) ${ }^{14}$ para testar o modelo de pesquisa e as hipóteses. SEM é um método estatístico que permite aos pesquisadores responderem a uma série de perguntas interrelacionadas de uma forma simples, sistemática e abrangente, por meio da modelagem das relações entre os construtos dependentes e independentes.

A Tabela 2 mostra os resultados dos testes de validade para o cenário, em que a consistência interna dos construtos foi avaliada quanto à sua confiabilidade. Os valores Alfa de Cronbach dos construtos foram próximos ao limite de

\begin{tabular}{|l|l|l|l|l|}
\hline Participantes & Construtos & $\begin{array}{l}\text { Número } \\
\text { de Itens }\end{array}$ & Média & $\begin{array}{l}\text { Alpha de } \\
\text { Cronbach }\end{array}$ \\
\hline $\begin{array}{l}\text { Cuidadores } \\
\text { de Saúde }\end{array}$ & $\begin{array}{l}\text { Facilidade de Uso } \\
\text { Percebida }\end{array}$ & 4 & 4.05 & 0.813 \\
\cline { 2 - 5 } & Utilidade Percebida & 5 & 4.50 & 0.851 \\
\cline { 2 - 5 } & Intenção de Uso & 3 & 4.39 & 0.799 \\
\hline \multirow{5}{*}{ Pacientes } & $\begin{array}{l}\text { Facilidade de Uso } \\
\text { Percebida }\end{array}$ & 4 & 4.13 & 0.790 \\
\cline { 2 - 6 } & Utilidade Percebida & 5 & 4.06 & 0.862 \\
\cline { 2 - 6 } & Intenção de Uso & 3 & 3.94 & 0.764 \\
\hline
\end{tabular}


0.700, que é considerado muito bom. Portanto, concluímos que esses construtos são confiáveis para serem utilizados em nossa análise de dados. Com base nesta análise, concluiu-se que os valores médios mostraram boa validade.

Em seguida, os dados validados foram analisados usando o modelo SEM. A intenção dos usuários em usar a aplicação para a troca de mensagens baseadas em arquétipos pode ser explicada ou prevista com base na facilidade de uso e utilidade percebida. Baseado nas hipóteses formuladas foi utilizado o modelo de pesquisa que indica os relacionamentos causais entre as variáveis do modelo TAM, considerando que o R2 é a porcentagem da variância total das variáveis independentes e indica a previsibilidade do modelo de pesquisa. As três hipóteses seguintes foram propostas e analisadas no cenário apresentado:

H1. Utilidade Percebida afeta positivamente a Intenção de Uso da aplicação.

- Hipótese Nula: H1n: $\mu_{\text {PerceivedUsefulness }}=\mu_{\text {IntentionUse }}$

- Hipótese Alternativa: H1a: $\mu_{\text {PerceivedUsefulness }} \neq \mu_{\text {IntentionUse }}$

H2. Facilidade de Uso Percebida afeta positivamente a Intenção de Uso da aplicação.

- Hipótese Nula: $\mathbf{H} 2 \boldsymbol{n}: \mu_{\text {PerceivedEaseUse }}=\mu_{\text {IntentionUse }}$

- Hipótese Alternativa: H2a: $\mu_{\text {PerceivedEaseUse }} \neq \mu_{\text {IntentionUse }}$

H3. Facilidade de Uso Percebida afeta positivamente a Utilidade Percebida.

- Hipótese Nula: H3n: $\mu_{\text {PerceivedEaseUse }}=\mu_{\text {PerceivedUsefulness }}$

- Hipótese Alternativa: H3a: $\mu_{\text {PerceivedEaseUse }} \neq \mu_{\text {PerceivedUsefulness }}$
Para verificar as três hipóteses foi utilizado o modelo de Análise de Regressão Estatística ${ }^{15}$ para os dados coletados de cuidadores e pacientes no cenário apresentado. Os resultados estão resumidos na Tabela 3. Por exemplo, no cenário (participantes=cuidadores), foi testada a hipótese H3a para verificar se a Utilidade Percebida é determinada pela Facilidade de Uso Percebida. Os detalhes da análise de regressão são: R2=0,61, $p=0,0005$ (alta importância para $p<0,001$ e $a=0,05$ ). Quanto aos resultados da regressão, pode-se rejeitar a hipótese nula (H3n), significando que foi confirmado empiricamente que Utilidade Percebida é determinada pela Facilidade de Uso Percebida, e que H3a foi fortemente confirmado. R2 indica que a Facilidade de Uso Percebida explica 61\% $(R 2=0.61)$ da variância na Utilidade Percebida. Hipóteses H1a e H2a foram confirmadas e a Utilidade Percebida determina fortemente a Intenção de Uso, enquanto a Facilidade de Uso Percebida foi um determinante secundário significativo.

Portanto, as três hipóteses foram confirmadas em todos os pontos de medição. Os cuidadores de saúde concluíram que a aplicação seria muito útil para as tarefas diárias e consideraram-na muito fácil de usar. A maioria dos pacientes identificaram alguns benefícios da usabilidade, tais como a eficiente troca de mensagem.

No entanto, a abordagem para a análise dos dados tem algumas limitações. Em primeiro lugar, o modelo de questionário não é completamente livre de subjetividade para cada entrevistado. Cada entrevistado reage de modo particular diante de um questionário. Em segundo lugar, foram agrupados todos os cuidadores de saúde e os resultados puderam ser generalizados, enquanto seria possível dividi-los em vários grupos.

\begin{tabular}{|c|c|c|c|c|c|c|c|c|}
\hline \multirow{4}{*}{ Tabela 3. Análise de } & & Hipóteses & H1 (F & $\rightarrow$ IU) & H2 (F & $\rightarrow$ IU) & H3 (F & $\rightarrow$ PU) \\
\hline & \multirow{3}{*}{$\begin{array}{l}\text { Cenário: } \\
\text { Avaliação de } \\
\text { Implante de } \\
\text { Marcapasso }\end{array}$} & Participantes & $\mathbf{R}^{2}$ & $p<0.001$ & $\mathbf{R}^{2}$ & $p<0.05$ & $\mathbf{R}^{2}$ & $p<0.001$ \\
\hline & & Caregivers & 0.72 & 0.0001 & 0.72 & 0.0009 & 0.61 & 0.0005 \\
\hline & & Patients & 0.35 & 0.0000 & 0.35 & 0.0007 & 0.55 & 0.0002 \\
\hline
\end{tabular}


Em terceiro lugar, outros fatores podem afetar a decisão das pessoas quanto ao uso de uma determinada tecnologia, como por exemplo, a experiência prévia e a relevância da tecnologia no trabalho ${ }^{16}$, os quais não foram levados em consideração no presente trabalho. No entanto, neste estudo consideramos Utilidade Percebida e Facilidade de Uso Percebida como os fatores mais importantes para explicar a futura intenção de utilizar a aplicação para troca de mensagens baseadas em arquétipos openEHR.

\section{Trabalhos Relacionados}

Devido principalmente à complexidade que envolve o processo de desenvolvimento de mensagens openEHR e à falta de ferramentas de suporte, várias propostas para facilitar o uso de padrões podem ser encontradas na literatura.

$\mathrm{Em}^{17}$, há uma discussão sobre a implementação de uma ferramenta de modelagem para troca de mensagens HL7, desenvolvida a partir da IDE Eclipse. Para lidar com os artefatos, a ferramenta desenvolvida permite a serialização das mensagens no padrão XML Metadata Interchange (XMI) para uso posterior.

$\mathrm{Em}^{18}$, é apresentada uma abordagem para desenvolvimento de sistema de informações laboratorial usando arquétipos para melhorar o processo de desenvolvimento de software.

$\mathrm{Em}^{19}$, há uma discussão sobre a implementação de uma fábrica de software focada no domínio da saúde. O objetivo de desenvolver a fábrica de software foi automatizar a criação de interfaces de comunicação, denominadas "portas de colaboração", a fim de facilitar a troca de mensagens.

$\mathrm{Em}^{20}$, é apresentada uma solução para auxiliar os desenvolvedores de software na manipulação de mensagens. A proposta dos autores é baseada na utilização de uma DSL programática, entre outras características, para suportar a criação de mensagens, transmissão, interpretação e validação.

A abordagem proposta neste trabalho baseia-se em diversas características dos trabalhos descritos acima. No entanto, apresenta suas próprias contribuições por meio da evolução e a adaptação dos conceitos descritos em trabalhos relacionados. Entre as contribuições deste trabalho, há a utilização de arquétipos para representar conceitos clínicos, que são integrados com mensagens openEHR. Uma DSL é também utilizada e se baseia em um metamodelo do domínio de saúde, que permite a geração de código automática e modelagem das interfaces de comunicação dos aplicativos nesse domínio.

\section{Conclusões}

Este trabalho apresentou uma abordagem para desenvolver aplicações no Ambiente de Cuidado de Saúde Pervasivo, e essa abordagem integra arquétipos a mensagens openEHR via uso de DSL. A integração dos arquétipos a mensagens openEHR possibilita agilidade e eficiência na comunicação entre sistemas de comunicação heterogêneos, uma vez que a comunicação se torna interoperável e conceitos clínicos podem ser facilmente identificados. O uso de DSLs permite a criação de uma infraestrutura para geração de código dessas mensagens e sua reutilização em diferentes aplicações no domínio de destino, o que reduz o esforço necessário para desenvolvimento de aplicação.

Realizamos um estudo abrangente para investigar a utilidade e a facilidade de uso de tecnologias no cuidado de saúde pervasivo dentro de ambientes de cuidado de saúde. Foi apresentado o cenário que mostra a aceitação da abordagem proposta por cuidadores de saúde e pacientes, que reagiram positivamente em relação à utilidade da abordagem na construção de aplicações. 
Na presente abordagem foi mostrada a geração parcial de código para manipular mensagens openEHR usando arquétipos por meio de transformações M2C.

Em trabalhos futuros, será avaliado o desempenho da abordagem proposta, especialmente com relação à escalabilidade, que é um requisito não-funcional crucial para desenvolvimento de aplicações realísticas e para o apoio simultâneo em múltiplos cenários. Planejamos futuros experimentos para estender o modelo TAM para investigar o efeito de outras variáveis - tais como, efeito da experiência prévia do usuário, qualidade das informações processadas e relevância do uso da tecnologia - na utilidade percebida, facilidade de uso percebida e a intenção de utilizar determinada tecnologia.

\section{Referências}

1. Varshney U. Pervasive Healthcare Computing - EMR/EHR, Wireless and Health Monitoring. New York: Springer; 2009.

2. Hansmann U, Merk L, Nicklous M, Stober T. Pervasive Computing : The Mobile World. 2nd Edition ed: Springer; 2003. $468 \mathrm{p}$.

3. Weiser M. Some Computer Science Issues in Ubiquitous Computing. Commun ACM. 1993;36(7):75-84. doi: 10.1145/159544.159617.

4. Browne E. openEHR Archetypes for HL7 CDA Documents. Ocean Informatics, 2008 Contract No.: 07 Dec 2012.

5. Mernik M, Heering J, Sloane AM. When and how to develop domain-specific languages. ACM Comput Surv. 2005;37(4):316-44. doi: 10.1145/1118890.1118892.

6. Beale T, Heard S. Archetype Definition Language. openEHR Foundation, 2007.

7. Deursen Av, Klint P, Visser J. Domain-specific languages: an annotated bibliography. SIGPLAN Not. 2000;35(6):26-36. doi: 10.1145/352029.352035.

8. Ross DT. Structured Analysis (SA): A Language for Communicating Ideas. Software Engineering, IEEE Transactions on. 1977;SE-3(1):16-34. doi: 10.1109/TSE.1977.229900.

9. Hevner AR, March ST, Park J, Ram S. Design Science in Information Systems Research. MIS Quarterly. 2004;28(1):75-105. PubMed PMID: WOS:000220207500005.

10. Davis FD. Perceived Usefulness, Perceived Ease of Use, and User Acceptance of Information Technology. MIS Q. 1989;13(3):319-40. doi: 10.2307/249008.

11. Chau PYK, Hu PJH. Investigating Healthcare Professionals' Decisions to Accept Telemedicine Technology: an Empirical Test of Competing Theories. Information \&amp; Management. 2002;39(4):297-311. doi: 10.1016/s0378-7206(01)00098-2. PubMed PMID: WOS:000173105000005.

12. Likert R. A Technique for the Measurement of Attitudes. New York, 1932. 55 p.

13. Nunnally JC. Psychometric Theory. Current Contents/Social \&amp; Behavioral Sciences. 1979(22). PubMed PMID: WOS:A1979HZ31300001.

14. Henseler J, Chin WW. A Comparison of Approaches for the Analysis of Interaction Effects Between Latent Variables Using Partial Least Squares Path Modeling. Structural Equation Modeling-a Multidisciplinary Journal. 2010;17(1):82-109. doi: 10.1080/10705510903439003. PubMed PMID: WOS:000273611500005.

15. Goldin RF. Review: Statistical Models-Theory and Practice. The American Mathematical Monthly. 2010;117(9):844-7. doi: 10.4169/000298910X521733.

16. Davis FD, Venkatesh V. Toward Preprototype User Acceptance Testing of New Information Systems: Implications for Software Project Management. IEEE Transactions on Engineering Management. 2004;51(1):31-46. doi: 10.1109/tem.2003.822468. PubMed PMID: WOS:000188950500004.

17. Banfai B, Ulrich B, Torok Z, Natarajan R, Ireland T. Implementing an HL7 version 3 modeling tool from an Ecore model. Studies in health technology and informatics. 2009;150:157-61. PubMed PMID: MEDLINE:19745289.

18. Piho G, Tepandi J, Parman M, Perkins D, editors. From archetypes-based domain model of clinical laboratory to LIMS software. MIPRO, 2010 Proceedings of the 33rd International Convention; 2010 24-28 May 2010.

19. Regio M, Greenfield J. Designing and Implementing an HL7 Software Factory. 2005. PubMed PMID: 243.

20. Ohr C, Václavík M, editors. Using HL7 Processing Capabilities of the Open Ehealth Integration Platform in the Implementation of IHE Profiles. ICW Developer Conference; 2009; Walldorf.

\footnotetext{
[1] http://www.hl7.org

[2] http://www.openehr.org

[3] http://www.oceaninformatics.com/

[4] http://www.ihtsdo.org/snomed-ct/
} 